\title{
Double sequence spaces over $n$-normed spaces defined by a sequence of Orlicz functions
}

\author{
Abdullah Alotaibi ${ }^{1}$, Mohammad Mursaleen ${ }^{2 *}$ and Sunil K Sharma ${ }^{3}$
}

\section{"Correspondence:}

mursaleenm@gmail.com

${ }^{2}$ Department of Mathematics,

Aligarh Muslim University, Aligarh,

202002, India

Full list of author information is

available at the end of the article

\begin{abstract}
In the present paper we introduce double sequence space $m^{2}(\mathcal{M}, A, \phi, p,\|\cdot, \ldots, \cdot\|)$ defined by a sequence of Orlicz functions over $n$-normed space. We examine some of its topological properties and establish some inclusion relations.

MSC: 40A05; 46A45

Keywords: double sequence spaces; paranormed space; Orlicz function; $n$-normed space
\end{abstract}

\section{Introduction and preliminaries}

The initial works on double sequences is found in Bromwich [1]. Later on, it was studied by Hardy [2], Moricz [3], Moricz and Rhoades [4], Başarır and Sonalcan [5] and many others. Hardy [2] introduced the notion of regular convergence for double sequences. Quite recently, Zeltser [6] in her PhD thesis has essentially studied both the theory of topological double sequence spaces and the theory of summability of double sequences. Mursaleen and Edely [7] have recently introduced the statistical convergence which was further studied in locally solid Riesz spaces [8]. Nextly, Mursaleen [9] and Mursaleen and Savas [10] have defined the almost regularity and almost strong regularity of matrices for double sequences and applied these matrices to establish core theorems and introduced the $M$-core for double sequences and determined those four dimensional matrices transforming every bounded double sequences $x=\left(x_{k, l}\right)$ into one whose core is a subset of the $M$-core of $x$. More recently, Altay and Başar [11] have defined the spaces $\mathcal{B S}, \mathcal{B S}(t), \mathcal{C S}_{p}, \mathcal{C S}_{b p}, \mathcal{C S}_{r}$ and $\mathcal{B V}$ of double sequences consisting of all double series whose sequence of partial sums are in the spaces $\mathcal{M}_{u}, \mathcal{M}_{u}(t), \mathcal{C}_{p}, \mathcal{C}_{b p}, \mathcal{C}_{r}$ and $\mathcal{L}_{u}$, respectively and also examined some properties of these sequence spaces and determined the $\alpha$-duals of the spaces $\mathcal{B S}, \mathcal{B V}, \mathcal{C} \mathcal{S}_{b p}$ and the $\beta(v)$-duals of the spaces $\mathcal{C} \mathcal{S}_{b p}$ and $\mathcal{C} \mathcal{S}_{r}$ of double series. Recently Başar and Sever [12] have introduced the Banach space $\mathcal{L}_{q}$ of double sequences corresponding to the well known space $\ell_{q}$ of single sequences and examined some properties of the space $\mathcal{L}_{q}$. Now, recently Raj and Sharma [13] have introduced entire double sequence spaces. By the convergence of a double sequence we mean the convergence in the Pringsheim sense i.e. a double sequence $x=\left(x_{k, l}\right)$ has Pringsheim limit $L$ (denoted by $P$ - $\left.\lim x=L\right)$ provided that given $\epsilon>0$ there exists $n \in N$ such that $\left|x_{k, l}-L\right|<\epsilon$ whenever $k, l>n$, see [14]. The double

\section{第 Springer}

(O2014 Alotaibi et al.: licensee Springer. This is an Open Access article distributed under the terms of the Creative Commons Attribution License (http://creativecommons.org/licenses/by/2.0), which permits unrestricted use, distribution, and reproduction in any medium, provided the original work is properly cited. 
sequence $x=\left(x_{k, l}\right)$ is bounded if there exists a positive number $M$ such that $\left|x_{k, l}\right|<M$ for all $k$ and $l$.

Throughout this paper, $\mathbb{N}$ and $\mathbb{C}$ denote the set of positive integers and complex numbers, respectively. A complex double sequence is a function $x$ from $N \times \mathbb{N}$ into $\mathbb{C}$ and briefly denoted by $\left\{x_{k, l}\right\}$. If for all $\epsilon>0$, there is $n_{\epsilon} \in \mathbb{N}$ such that $\left|x_{k, l}-a\right|<\epsilon$ where $k>n_{\epsilon}$ and $l>n_{\epsilon}$, then a double sequence $\left\{x_{k, l}\right\}$ is said to be convergent to $a \in \mathbb{C}$. A real double sequence $\left\{x_{k, l}\right\}$ is non-decreasing, if $x_{k, l} \leq x_{p, q}$ for $(k, l)<(p, q)$. A double series is infinite sum $\sum_{k, l=1}^{\infty} x_{k, l}$ and its convergence implies the convergence of partial sums sequence $\left\{S_{n, m}\right\}$, where $S_{n, m}=\sum_{k=1}^{m} \sum_{l=1}^{n} x_{k, l}$ (see [15]). For recent development on double sequences, we refer to [16-20] and [21-23].

A double sequence space $E$ is said to be solid if $\left\{x_{k, l} y_{k, l}\right\} \in E$ for all double sequences $\left\{y_{k, l}\right\}$ of scalars such that $\left|y_{k, l}\right|<1$ for all $k, l \in N$ whenever $\left\{x_{k, l}\right\} \in E$.

Let $x=\left\{x_{k, l}\right\}$ be a double sequence. A set $S(x)$ is defined by

$$
S(x)=\left\{\left\{X_{\pi_{1}(k), \pi_{2}(k)}\right\}: \pi_{1} \text { and } \pi_{2} \text { are permutation of } \mathbb{N}\right\} .
$$

If $S(x) \subseteq E$ for all $x \in E$, then $E$ is said to be symmetric. Now let $\mathcal{P}_{s}$ be a family of subsets $\sigma$ having at most elements $s$ in $\mathbb{N}$. Also $\mathcal{P}_{s, t}$ denotes the class of subsets $\sigma=\sigma_{1} \times \sigma_{2}$ in $\mathbb{N} \times \mathbb{N}$ such that the element numbers of $\sigma_{1}$ and $\sigma_{2}$ are at most $s$ and $t$, respectively. Besides $\left\{\phi_{k, l}\right\}$ is taken as a non-decreasing double sequence of the positive real numbers such that

$$
\begin{aligned}
k \phi_{k+1, l} & \leq(k+1) \phi_{k, l}, \\
l \phi_{k, l+1} & \leq(l+1) \phi_{k, l} .
\end{aligned}
$$

An Orlicz function $M:[0, \infty) \rightarrow[0, \infty)$ is a continuous, non-decreasing, and convex function such that $M(0)=0, M(x)>0$ for $x>0$ and $M(x) \rightarrow \infty$ as $x \rightarrow \infty$.

Lindenstrauss and Tzafriri [24] used the idea of Orlicz function to define the following sequence space:

$$
\ell_{M}=\left\{x \in w: \sum_{k=1}^{\infty} M\left(\frac{\left|x_{k}\right|}{\rho}\right)<\infty\right\},
$$

which is called an Orlicz sequence space. Also $\ell_{M}$ is a Banach space with the norm

$$
\|x\|=\inf \left\{\rho>0: \sum_{k=1}^{\infty} M\left(\frac{\left|x_{k}\right|}{\rho}\right) \leq 1\right\} .
$$

Also, it was shown that every Orlicz sequence space $\ell_{M}$ contains a subspace isomorphic to $\ell_{p}(p \geq 1)$. The $\Delta_{2}$-condition is equivalent to $M(L x) \leq L M(x)$, for all $L$ with $0<L<1$. An Orlicz function $M$ can always be represented in the following integral form:

$$
M(x)=\int_{0}^{x} \eta(t) d t
$$

where $\eta$, known as the kernel of $M$, is right differentiable for $t \geq 0, \eta(0)=0, \eta(t)>0, \eta$ is non-decreasing and $\eta(t) \rightarrow \infty$ as $t \rightarrow \infty$. 
For further reading on Orlicz spaces, we refer to [25-29].

Let $X$ be a linear metric space. A function $p: X \rightarrow \mathbb{R}$ is called a paranorm if

(1) $p(x) \geq 0$ for all $x \in X$,

(2) $p(-x)=p(x)$ for all $x \in X$,

(3) $p(x+y) \leq p(x)+p(y)$ for all $x, y \in X$,

(4) if $\left(\lambda_{n}\right)$ is a sequence of scalars with $\lambda_{n} \rightarrow \lambda$ as $n \rightarrow \infty$ and $\left(x_{n}\right)$ is a sequence of vectors with $p\left(x_{n}-x\right) \rightarrow 0$ as $n \rightarrow \infty$, then $p\left(\lambda_{n} x_{n}-\lambda x\right) \rightarrow 0$ as $n \rightarrow \infty$.

A paranorm $p$ for which $p(x)=0$ implies $x=0$ is called a total paranorm and the pair $(X, p)$ is called a total paranormed space. It is well known that the metric of any linear metric space is given by some total paranorm (see [30], Theorem 10.4.2, p.183).

The concept of 2-normed spaces was initially developed by Gähler [31] in the mid-1960s, while that of $n$-normed spaces one can see in Misiak [32]. Since then, many others have studied this concept and obtained various results; see Gunawan [33, 34] and Gunawan and Mashadi [35] and references therein. Let $n \in \mathbb{N}$ and $X$ be a linear space over the field $\mathbb{K}$, where $\mathbb{K}$ is the field of real or complex numbers of dimension $d$, where $d \geq n \geq 2$. A real valued function $\|\cdot, \ldots, \cdot\|$ on $X^{n}$ satisfying the following four conditions:

(1) $\left\|x_{1}, x_{2}, \ldots, x_{n}\right\|=0$ if and only if $x_{1}, x_{2}, \ldots, x_{n}$ are linearly dependent in $X$;

(2) $\left\|x_{1}, x_{2}, \ldots, x_{n}\right\|$ is invariant under permutation;

(3) $\left\|\alpha x_{1}, x_{2}, \ldots, x_{n}\right\|=|\alpha|\left\|x_{1}, x_{2}, \ldots, x_{n}\right\|$ for any $\alpha \in \mathbb{K}$, and

(4) $\left\|x+x^{\prime}, x_{2}, \ldots, x_{n}\right\| \leq\left\|x, x_{2}, \ldots, x_{n}\right\|+\left\|x^{\prime}, x_{2}, \ldots, x_{n}\right\|$

is called a $n$-norm on $X$, and the pair $(X,\|\cdot, \ldots, \cdot\|)$ is called a $n$-normed space over the field $\mathbb{K}$. For example, we may take $X=\mathbb{R}^{n}$ being equipped with the Euclidean $n$-norm $\left\|x_{1}, x_{2}, \ldots, x_{n}\right\|_{E}$, the volume of the $n$-dimensional parallelepiped spanned by the vectors $x_{1}, x_{2}, \ldots, x_{n}$ which may be given explicitly by the formula

$$
\left\|x_{1}, x_{2}, \ldots, x_{n}\right\|_{E}=\left|\operatorname{det}\left(x_{i j}\right)\right|
$$

where $x_{i}=\left(x_{i 1}, x_{i 2}, \ldots, x_{i n}\right) \in \mathbb{R}^{n}$ for each $i=1,2, \ldots, n$. Let $(X,\|\cdot, \ldots, \cdot\|)$ be a $n$-normed space of dimension $d \geq n \geq 2$ and $\left\{a_{1}, a_{2}, \ldots, a_{n}\right\}$ be linearly independent set in $X$. Then the function $\|\cdot, \ldots, \cdot\|_{\infty}$ on $X^{n-1}$ defined by

$$
\left\|x_{1}, x_{2}, \ldots, x_{n-1}\right\|_{\infty}=\max \left\{\left\|x_{1}, x_{2}, \ldots, x_{n-1}, a_{i}\right\|: i=1,2, \ldots, n\right\}
$$

defines an $(n-1)$-norm on $X$ with respect to $\left\{a_{1}, a_{2}, \ldots, a_{n}\right\}$.

A sequence $\left(x_{k}\right)$ in a $n$-normed space $(X,\|\cdot, \ldots, \cdot\|)$ is said to converge to some $L \in X$ if

$$
\lim _{k \rightarrow \infty}\left\|x_{k}-L, z_{1}, \ldots, z_{n-1}\right\|=0 \quad \text { for every } z_{1}, \ldots, z_{n-1} \in X
$$

A sequence $\left(x_{k}\right)$ in a $n$-normed space $(X,\|\cdot, \ldots, \cdot\|)$ is said to be Cauchy if

$$
\lim _{\substack{k \rightarrow \infty \\ p \rightarrow \infty}}\left\|x_{k}-x_{p}, z_{1}, \ldots, z_{n-1}\right\|=0 \quad \text { for every } z_{1}, \ldots, z_{n-1} \in X
$$

If every Cauchy sequence in $X$ converges to some $L \in X$, then $X$ is said to be complete with respect to the $n$-norm. Any complete $n$-normed space is said to be $n$-Banach space. 
The space $m(\phi)$ was introduced by Sargent [36]:

$$
m(\phi)=\left\{x=\left(x_{k}\right) \in w:\|x\|_{m(\phi)}=\sup _{s \geq 1, \sigma \in \mathcal{P}_{s}} \frac{1}{\phi_{s}} \sum_{k \in \sigma}\left|x_{k}\right|<\infty\right\},
$$

which was further studied in [37,38] and [39]. Recently, Duyar and Oǧur [40] introduced the sequence space $m^{2}(M, A, \phi, p)$ and studied some of its properties.

Let $A=\left(a_{i j k l}\right)$ be an infinite double matrix of complex numbers, $\mathcal{M}=\left(M_{k, l}\right)$ be a sequence of Orlicz functions, and $p=\left(p_{k, l}\right)$ be a bounded double sequence of positive real numbers. In the present paper we define the following sequence space:

$$
\begin{aligned}
& m^{2}(\mathcal{M}, A, \phi, p,\|\cdot \ldots, \cdot\|) \\
& =\left\{x=\left(x_{k, l}\right) \in w^{2}(X): \sup \left\{\frac{1}{\phi_{s, t}} \sum_{i \in \sigma_{1}} \sum_{j \in \sigma_{2}} \sum_{k, l=1}^{\infty} M_{k, l}\left(\left\|\frac{A_{i j}(x)}{\rho}, z_{1}, \ldots, z_{n-1}\right\|\right)^{p_{i j}}:\right.\right. \\
& \left.\left.\quad(s, t) \geq(1,1), \sigma_{1} \times \sigma_{2} \in \mathcal{P}_{s, t}\right\}<\infty \text { for some } \rho>0\right\},
\end{aligned}
$$

where $A(x)=\left(A_{i j}(x)\right)$ if $A_{i j}(x)=\sum_{k, l=1}^{\infty} a_{i j k l} x_{k, l}$ converges for each $(i, j) \in \mathbb{N} \times \mathbb{N}$.

If $p=\left(p_{i j}\right)=1$, we have

$$
\begin{aligned}
& m^{2}(\mathcal{M}, A, \phi,\|\cdot, \ldots, \cdot\|) \\
& =\left\{x=\left(x_{k, l}\right) \in w^{2}(X): \sup \left\{\frac{1}{\phi_{s, t}} \sum_{i \in \sigma_{1}} \sum_{j \in \sigma_{2}} \sum_{k, l=1}^{\infty} M_{k, l}\left(\left\|\frac{A_{i j}(x)}{\rho}, z_{1}, \ldots, z_{n-1}\right\|\right):\right.\right. \\
& \left.\left.\quad(s, t) \geq(1,1), \sigma_{1} \times \sigma_{2} \in \mathcal{P}_{s, t}\right\}<\infty \text { for some } \rho>0\right\} .
\end{aligned}
$$

The following inequality will be used throughout the paper:

$$
|a+b|^{p_{i j}} \leq \max \left(1,2^{H-1}\right)\left(|a|^{p_{i j}}+|b|^{p_{i j}}\right),
$$

where $a, b \in \mathbb{C}$ and $H=\sup \left\{p_{i j}:(i, j) \in \mathbb{N} \times \mathbb{N}\right\}$.

We examine some topological properties of $m^{2}(\mathcal{M}, A, \phi, p,\|\cdot, \ldots, \cdot\|)$ and establish some inclusion relations.

\section{Main results}

Theorem 2.1 Let $\mathcal{M}=\left(M_{k, l}\right)$ be a sequence of Orlicz functions and $p=\left(p_{k, l}\right)$ be a bounded sequence of positive real numbers, then the space $m^{2}(\mathcal{M}, A, \phi, p,\|\cdot \ldots, \cdot\|)$ is linear space over the field of complex number $\mathbb{C}$.

Proof Let $x=\left(x_{k, l}\right), y=\left(y_{k, l}\right) \in m^{2}(\mathcal{M}, A, \phi, p,\|\cdot, \ldots, \cdot\|)$ and $\alpha, \beta \in \mathbb{C}$. Then there exist positive numbers $\rho_{1}$ and $\rho_{2}$ such that

$$
\sup \left\{\frac{1}{\phi_{s, t}} \sum_{i \in \sigma_{1}} \sum_{j \in \sigma_{2}} \sum_{k, l=1}^{\infty} M_{k, l}\left(\left\|\frac{A_{i j}(x)}{\rho_{1}}, z_{1}, \ldots, z_{n-1}\right\|\right)^{p_{i j}}:(s, t) \geq(1,1), \sigma_{1} \times \sigma_{2} \in \mathcal{P}_{s, t}\right\}<\infty
$$


and

$$
\sup \left\{\frac{1}{\phi_{s, t}} \sum_{i \in \sigma_{1}} \sum_{j \in \sigma_{2}} \sum_{k, l=1}^{\infty} M_{k, l}\left(\left\|\frac{A_{i j}(y)}{\rho_{2}}, z_{1}, \ldots, z_{n-1}\right\|\right)^{p_{i j}}:(s, t) \geq(1,1), \sigma_{1} \times \sigma_{2} \in \mathcal{P}_{s, t}\right\}<\infty .
$$

Let $\rho_{3}=\max \left(2|\alpha| \rho_{1}, 2|\beta| \rho_{2}\right)$. Since $\mathcal{M}$ is a non-decreasing and convex function, we have

$$
\begin{aligned}
& \sum_{i \in \sigma_{1}} \sum_{j \in \sigma_{2}} \sum_{k, l=1}^{\infty} M_{k, l}\left(\left\|\frac{A_{i j}(\alpha x+\beta y)}{\rho_{3}}, z_{1}, \ldots, z_{n-1}\right\|\right)^{p_{i j}} \\
& \leq \sum_{i \in \sigma_{1}} \sum_{j \in \sigma_{2}} \sum_{k, l=1}^{\infty} M_{k, l}\left(\left\|\frac{\alpha a_{i j k l} x_{k, l}+\beta a_{i j k l} y_{k, l}}{\rho_{3}}, z_{1}, \ldots, z_{n-1}\right\|\right)^{p_{i j}} \\
& \leq \sum_{i \in \sigma_{1}} \sum_{j \in \sigma_{2}} \sum_{k, l=1}^{\infty} M_{k, l}\left(\left\|\frac{\alpha a_{i j k l} x_{k, l}}{2|\alpha| \rho_{1}}, z_{1}, \ldots, z_{n-1}\right\|+\left\|\frac{\beta a_{i j k l} y_{k, l}}{2|\beta| \rho_{2}}, z_{1}, \ldots, z_{n-1}\right\|\right)^{p_{i j}} \\
&= \sum_{i \in \sigma_{1}} \sum_{j \in \sigma_{2}} \sum_{k, l=1}^{\infty} M_{k, l}\left(\left\|\frac{a_{i j k l} x_{k, l}}{2 \rho_{1}}, z_{1}, \ldots, z_{n-1}\right\|+\left\|\frac{a_{i j k l} y_{k, l}}{2 \rho_{2}}, z_{1}, \ldots, z_{n-1}\right\|\right)^{p_{i j}} \\
& \leq \max \left(1,2^{H-1}\right)\left(\sum_{i \in \sigma_{1}} \sum_{j \in \sigma_{2}} \sum_{k, l=1}^{\infty} M_{k, l}\left(\left\|\frac{a_{i j k l} x_{k, l}}{2 \rho_{1}}, z_{1}, \ldots, z_{n-1}\right\|\right)^{p_{i j}}\right. \\
&\left.\quad+\sum_{i \in \sigma_{1}} \sum_{j \in \sigma_{2}} \sum_{k, l=1}^{\infty} M_{k, l}\left(\left\|\frac{a_{i j k l} y_{k, l}}{2 \rho_{2}}, z_{1}, \ldots, z_{n-1}\right\|\right)^{p_{i j}}\right) .
\end{aligned}
$$

Thus, we have

$$
\begin{aligned}
& \sup \left\{\frac{1}{\phi_{s, t}} \sum_{i \in \sigma_{1}} \sum_{j \in \sigma_{2}} \sum_{k, l=1}^{\infty} M_{k, l}\left(\left\|\frac{A_{i j}(\alpha x+\beta y)}{\rho_{3}}, z_{1}, \ldots, z_{n-1}\right\|\right)^{p_{i j}}:\right. \\
& \left.(s, t) \geq(1,1), \sigma_{1} \times \sigma_{2} \in \mathcal{P}_{s, t}\right\} \\
& \leq \max \left(1,2^{H-1}\right)\left\{\operatorname { s u p } \left\{\frac{1}{\phi_{s, t}} \sum_{i \in \sigma_{1}} \sum_{j \in \sigma_{2}} \sum_{k, l=1}^{\infty} M_{k, l}\left(\left\|\frac{a_{i j k l} x_{k, l}}{2 \rho_{1}}, z_{1}, \ldots, z_{n-1}\right\|\right)^{p_{i j}}:\right.\right. \\
& \left.\quad(s, t) \geq(1,1), \sigma_{1} \times \sigma_{2} \in \mathcal{P}_{s, t}\right\} \\
& \quad+\sup \left\{\frac{1}{\phi_{s, t}} \sum_{i \in \sigma_{1}} \sum_{j \in \sigma_{2}} M_{k, l}\left(\left\|\frac{a_{i j k l} y_{k, l}}{2 \rho_{2}}, z_{1}, \ldots, z_{n-1}\right\|\right)^{p_{i j}}:\right. \\
& \left.\left.(s, t) \geq(1,1), \sigma_{1} \times \sigma_{2} \in \mathcal{P}_{s, t}\right\}\right\} .
\end{aligned}
$$

This proves that $\alpha x+\beta y \in m^{2}(\mathcal{M}, A, \phi, p,\|\cdot, \ldots, \cdot\|)$. Hence $m^{2}(\mathcal{M}, A, \phi, p,\|\cdot, \ldots, \cdot\|)$ is a linear space. This completes the proof of the theorem.

Theorem 2.2 $\mathcal{M}=\left(M_{k, l}\right)$ be a sequence of Orlicz functions and $p=\left(p_{k, l}\right)$ be a bounded sequence of positive real numbers, then the space $m^{2}(\mathcal{M}, A, \phi, p,\|\cdot, \ldots, \cdot\|)$ is a paranormed 
space with the paranorm defined by

$$
\begin{gathered}
g(x)=\inf \left\{\rho^{\frac{p_{q r}}{H}}:\left[\operatorname { s u p } \left\{\frac{1}{\phi_{s, t}} \sum_{i \in \sigma_{1}} \sum_{j \in \sigma_{2}} \sum_{k, l=1}^{\infty} M_{k, l}\left(\left\|\frac{A_{i j}(x)}{\rho}, z_{1}, \ldots, z_{n-1}\right\|\right)^{p_{i j}}:\right.\right.\right. \\
\left.\left.\left.(s, t) \geq(1,1), \sigma_{1} \times \sigma_{2} \in \mathcal{P}_{s, t}\right\} \leq 1\right]^{1 / H}, q \in \mathbb{N}, r \in \mathbb{N}\right\} .
\end{gathered}
$$

Proof It is clear that $g(x)=g(-x)$ and $g(x)=0$ if $x=0$. Then there exist positive numbers $\rho_{1}$ and $\rho_{2}$ such that

$$
\sup \left\{\frac{1}{\phi_{s, t}} \sum_{i \in \sigma_{1}} \sum_{j \in \sigma_{2}} \sum_{k, l=1}^{\infty} M_{k, l}\left(\left\|\frac{A_{i j}(x)}{\rho_{1}}, z_{1}, \ldots, z_{n-1}\right\|\right)^{p_{i j}}:(s, t) \geq(1,1), \sigma_{1} \times \sigma_{2} \in \mathcal{P}_{s, t}\right\}<1
$$

and

$$
\sup \left\{\frac{1}{\phi_{s, t}} \sum_{i \in \sigma_{1}} \sum_{j \in \sigma_{2}} \sum_{k, l=1}^{\infty} M_{k, l}\left(\left\|\frac{A_{i j}(y)}{\rho_{2}}, z_{1}, \ldots, z_{n-1}\right\|\right)^{p_{i j}}:(s, t) \geq(1,1), \sigma_{1} \times \sigma_{2} \in \mathcal{P}_{s, t}\right\}<1 .
$$

Then, by using Minkowski's inequality, we have

$$
\begin{aligned}
\sup \{ & \frac{1}{\phi_{s, t}} \sum_{i \in \sigma_{1}} \sum_{j \in \sigma_{2}} \sum_{k, l=1}^{\infty} M_{k, l}\left(\left\|\frac{A_{i j}(x+y)}{\rho_{1}+\rho_{2}}, z_{1}, \ldots, z_{n-1}\right\|\right)^{p_{i j}}: \\
& \left.(s, t) \geq(1,1), \sigma_{1} \times \sigma_{2} \in \mathcal{P}_{s, t}\right\} \\
\leq & \sup \left\{\frac { 1 } { \phi _ { s , t } } \sum _ { i \in \sigma _ { 1 } } \sum _ { j \in \sigma _ { 2 } } \sum _ { k , l = 1 } ^ { \infty } M _ { k , l } \left(\left\|\frac{A_{i j}(x)}{\rho_{1}+\rho_{2}}, z_{1}, \ldots, z_{n-1}\right\|\right.\right. \\
& \left.\left.+\left\|\frac{A_{i j}(y)}{\rho_{1}+\rho_{2}}, z_{1}, \ldots, z_{n-1}\right\|\right)^{p_{i j}}:(s, t) \geq(1,1), \sigma_{1} \times \sigma_{2} \in \mathcal{P}_{s, t}\right\} \\
\leq & \left.\frac{\rho_{1}}{\rho_{1}+\rho_{2}}\right)^{h}\left\{\frac{1}{\phi_{s, t}} \sum_{i \in \sigma_{1}} \sum_{j \in \sigma_{2}} \sum_{k, l=1}^{\infty} M_{k, l}\left(\left\|\frac{A_{i j}(x)}{\rho_{1}}, z_{1}, \ldots, z_{n-1}\right\|\right)^{p_{i j}}:\right. \\
& \left.(s, t) \geq(1,1), \sigma_{1} \times \sigma_{2} \in \mathcal{P}_{s, t}\right\} \\
& +\left(\frac{\rho_{2}}{\rho_{1}+\rho_{2}}\right)^{h}\left\{\frac{1}{\phi_{s, t}} \sum_{i \in \sigma_{1}} \sum_{j \in \sigma_{2}} \sum_{k, l=1}^{\infty} M_{k, l}\left(\left\|\frac{A_{i j}(y)}{\rho_{2}}, z_{1}, \ldots, z_{n-1}\right\|\right)^{p_{i j}}:\right. \\
& \left.(s, t) \geq(1,1), \sigma_{1} \times \sigma_{2} \in \mathcal{P}_{s, t}\right\},
\end{aligned}
$$

where $h=\inf p_{i j}$. This shows that $g(x+y) \leq g(x)+g(y)$. Using this triangle inequality we can write

$$
g\left(\lambda^{n} x^{n}-\lambda x\right) \leq g\left(\lambda^{n} x^{n}-\lambda^{n} x\right)+g\left(\lambda^{n} x-\lambda x\right)
$$


Alotaibi et al. Journal of Inequalities and Applications 2014, 2014:216

Page 7 of 12

http://www.journalofinequalitiesandapplications.com/content/2014/1/216

Thus we have

$$
\begin{aligned}
& g\left(\lambda^{n} x^{n}-\lambda^{n} x\right) \\
& =\inf \left\{\rho_{n}^{\frac{p_{q r}}{H}}:\left[\operatorname { s u p } \left\{\frac{1}{\phi_{s, t}} \sum_{i \in \sigma_{1}} \sum_{j \in \sigma_{2}} \sum_{k, l=1}^{\infty} M_{k, l}\left(\left\|\frac{A_{i j}\left(\lambda^{n} x^{n}-\lambda^{n} x\right)}{\rho_{n}}, z_{1}, \ldots, z_{n-1}\right\|\right)^{p_{i j}}:\right.\right.\right. \\
& \left.\left.\left.(s, t) \geq(1,1), \sigma_{1} \sigma_{2} \in \mathcal{P}_{s, t}\right\} \leq 1\right]^{1 / H} \leq 1, q \in \mathbb{N}, r \in \mathbb{N}\right\} \\
& =\inf \left\{\rho_{n}^{p_{q r} / H}:\left[\operatorname { s u p } \left\{\frac{1}{\phi_{s, t}} \sum_{i \in \sigma_{1}} \sum_{j \in \sigma_{2}} \sum_{k, l=1}^{\infty} M_{k, l}\left(\left\|\frac{A_{i j}\left(x^{n}-x\right)}{\left(\rho_{n} /\left|\lambda^{n}\right|\right)}, z_{1}, \ldots, z_{n-1}\right\|\right)^{p_{i j}}:\right.\right.\right. \\
& \left.\left.\left.(s, t) \geq(1,1), \sigma_{1} \times \sigma_{2} \in \mathcal{P}_{s, t}\right\} \leq 1\right]^{1 / H} \leq 1, q \in \mathbb{N}, r \in \mathbb{N}\right\} \\
& =\inf \left\{\left(\lambda^{n} \rho_{n}\right)^{p_{q r} / H}:\left[\operatorname { s u p } \left\{\frac{1}{\phi_{s, t}} \sum_{i \in \sigma_{1}} \sum_{j \in \sigma_{2}} \sum_{k, l=1}^{\infty} M_{k, l}\left(\left\|\frac{A_{i j}\left(x^{n}-x\right)}{\rho_{n}}, z_{1}, \ldots, z_{n-1}\right\|\right)^{p_{i j}}:\right.\right.\right. \\
& \left.\left.\left.(s, t) \geq(1,1), \sigma_{1} \times \sigma_{2} \in \mathcal{P}_{s, t}\right\}\right]^{1 / H} \leq 1, q \in \mathbb{N}, r \in \mathbb{N}\right\} \\
& \leq \max \left\{\left|\lambda^{n}\right|^{h / H},\left|\lambda^{n}\right|\right\} \times \inf \left\{\left(\left|\lambda^{n}\right| \rho_{n}\right)^{p_{q r} / H}:\right. \\
& {\left[\operatorname { s u p } \left\{\frac{1}{\phi_{s, t}} \sum_{i \in \sigma_{1}} \sum_{j \in \sigma_{2}} \sum_{k, l=1}^{\infty} M_{k, l}\left(\left\|\frac{A_{i j}\left(x^{n}-x\right)}{\rho_{n}}, z_{1}, \ldots, z_{n-1}\right\|\right)^{p_{i j}}:\right.\right.} \\
& \left.\left.\left.(s, t) \geq(1,1), \sigma_{1} \times \sigma_{2} \in \mathcal{P}_{s, t}\right\}\right]^{1 / H} \leq 1, q \in \mathbb{N}, r \in \mathbb{N}\right\} \\
& =\max \left\{\left|\lambda^{n}\right|^{h / H},\left|\lambda^{n}\right|\right\} \cdot g\left(x^{n}-x\right) .
\end{aligned}
$$

Thus

$$
\begin{aligned}
g( & \left.\lambda^{n} x-\lambda x\right) \\
= & \inf \left\{\rho_{n}^{p_{q r} / H}:\left[\operatorname { s u p } \left\{\frac{1}{\phi_{s, t}} \sum_{i \in \sigma_{1}} \sum_{j \in \sigma_{2}} \sum_{k, l=1}^{\infty} M_{k, l}\left(\left\|\frac{A_{i j}\left(\left(\lambda^{n}-\lambda\right) x\right)}{\rho_{n}}, z_{1}, \ldots, z_{n-1}\right\|\right)^{p_{i j}}:\right.\right.\right. \\
& \left.\left.\left.(s, t) \geq(1,1), \sigma_{1} \times \sigma_{2} \in \mathcal{P}_{s, t}\right\}\right]^{1 / H} \leq 1, q \in \mathbb{N}, r \in \mathbb{N}\right\} \\
= & \inf \left\{\rho_{n}^{p_{q r} / H}:\left[\operatorname { s u p } \left\{\frac{1}{\phi_{s, t}} \sum_{i \in \sigma_{1}} \sum_{j \in \sigma_{2}} \sum_{k, l=1}^{\infty} M_{k, l}\left(\left\|\frac{A_{i j}(x)}{\rho_{n} /\left|\lambda^{n}-\lambda\right|}, z_{1}, \ldots, z_{n-1}\right\|\right)^{p_{i j}}:\right.\right.\right. \\
& \left.\left.\left.(s, t) \geq(1,1), \sigma_{1} \times \sigma_{2} \in \mathcal{P}_{s, t}\right\}\right]^{1 / H} \leq 1, q \in \mathbb{N}, r \in \mathbb{N}\right\} \\
= & \inf \left\{\left(\left|\lambda^{n}-\lambda\right| \rho_{n}\right)^{p_{q r} / H}:\left[\operatorname { s u p } \left\{\frac{1}{\phi_{s, t}} \sum_{i \in \sigma_{1}} \sum_{j \in \sigma_{2}} \sum_{k, l=1}^{\infty} M_{k, l}\left(\left\|\frac{A_{i j}(x)}{\rho_{n}}, z_{1}, \ldots, z_{n-1}\right\|\right)^{p_{i j}}:\right.\right.\right.
\end{aligned}
$$




$$
\begin{aligned}
& \left.\left.\left.(s, t) \geq(1,1), \sigma_{1} \times \sigma_{2} \in \mathcal{P}_{s, t}\right\}\right]^{1 / H} \leq 1, q \in \mathbb{N}, r \in \mathbb{N}\right\} \\
\leq & \max \left\{\left|\lambda^{n}-\lambda\right|^{h / H},\left|\lambda^{n}-\lambda\right|\right\} g(x) .
\end{aligned}
$$

Hence $g\left(\lambda^{n} x^{n}-\lambda x\right) \rightarrow 0$ where $\lambda^{n} \rightarrow \lambda$ and $x^{n} \rightarrow x$ as $n \rightarrow \infty$. This proves that $m^{2}(\mathcal{M}, A, \phi, p,\|\cdot, \ldots, \cdot\|)$ is a paranormed space with the paranorm defined by $g$. This completes the proof of the theorem.

Theorem 2.3 Let $\phi$ and $\psi$ be two double sequences then $m^{2}(\mathcal{M}, A, \phi, p,\|\cdot, \ldots, \cdot\|) \subseteq$ $m^{2}(\mathcal{M}, A, \psi, p,\|\cdot, \ldots, \cdot\|)$ if and only if $\sup _{(s, t) \geq(1,1)}\left(\phi_{s, t} / \psi_{s, t}\right)<\infty$.

Proof Let $K=\sup _{(s, t) \geq(1,1)}\left(\phi_{s, t} / \psi_{s, t}\right)<\infty$. Then $\phi_{s, t} \leq K \cdot \psi_{s, t}$ for all $(s, t) \geq(1,1)$. If $x=$ $\left\{x_{k, l}\right\} \in m^{2}(\mathcal{M}, A, \phi, p,\|\cdot, \ldots, \cdot\|)$, then

$$
\begin{aligned}
& \sup \left\{\frac{1}{\phi_{s, t}} \sum_{i \in \sigma_{1}} \sum_{j \in \sigma_{2}} \sum_{k, l=1}^{\infty} M_{k, l}\left(\left\|\frac{A_{i j}(x)}{\rho}, z_{1}, \ldots, z_{n-1}\right\|\right)^{p_{i j}}:(s, t) \geq(1,1), \sigma_{1} \times \sigma_{2} \in \mathcal{P}_{s, t}\right\} \\
& \quad<\infty \text { for some } \rho>0 .
\end{aligned}
$$

Thus

$$
\begin{aligned}
& \sup \left\{\frac{1}{K \psi_{s, t}} \sum_{i \in \sigma_{1}} \sum_{j \in \sigma_{2}} \sum_{k, l=1}^{\infty} M_{k, l}\left(\left\|\frac{A_{i j}(x)}{\rho}, z_{1}, \ldots, z_{n-1}\right\|\right)^{p_{i j}}:(s, t) \geq(1,1), \sigma_{1} \times \sigma_{2} \in \mathcal{P}_{s, t}\right\} \\
& <\infty \text { for some } \rho>0
\end{aligned}
$$

and hence $x=\left\{x_{k, l}\right\} \in m^{2}(\mathcal{M}, A, \psi, p,\|\cdot, \ldots, \cdot\|)$. This shows that

$$
m^{2}(\mathcal{M}, A, \phi, p,\|\cdot, \ldots, \cdot\|) \subseteq m^{2}(\mathcal{M}, A, \psi, p,\|\cdot, \ldots, \cdot\|)
$$

Conversely, let $m^{2}(\mathcal{M}, A, \phi, p,\|\cdot, \ldots, \cdot\|) \subseteq m^{2}(\mathcal{M}, A, \psi, p,\|\cdot, \ldots, \cdot\|)$ and $\alpha_{s, t}=\frac{\phi_{s, t}}{\psi_{s, t}}$ for all $(s, t) \geq(1,1)$, and suppose $\sup _{(s, t) \geq(1,1)} \alpha_{s, t}=\infty$. Then there exists a subsequence $\left\{\alpha_{s_{i}, t_{i}}\right\}$ of $\left\{\alpha_{s, t}\right\}$ such that $\lim _{i \rightarrow \infty} \alpha_{s_{i}, t_{i}}=\infty$. If $x=\left\{x_{k, l}\right\} \in m^{2}(\mathcal{M}, A, \phi, p,\|\cdot, \ldots, \cdot\|)$, then we have

$$
\begin{gathered}
\sup \left\{\frac{1}{\psi_{s, t}} \sum_{i \in \sigma_{1}} \sum_{j \in \sigma_{2}} \sum_{k, l=1}^{\infty} M_{k, l}^{\infty}\left(\left\|\frac{A_{i j}(x)}{\rho}, z_{1}, \ldots, z_{n-1}\right\|\right)^{p_{i j}}:(s, t) \geq(1,1), \sigma_{1} \times \sigma_{2} \in \mathcal{P}_{s, t}\right\} \\
=\sup \left\{\alpha_{s, t} \frac{1}{\phi_{s, t}} \sum_{i \in \sigma_{1}} \sum_{j \in \sigma_{2}} \sum_{k, l=1}^{\infty} M_{k, l}^{\infty}\left(\left\|\frac{A_{i j}(x)}{\rho}, z_{1}, \ldots, z_{n-1}\right\|\right)^{p_{i j}}:\right. \\
\left.(s, t) \geq(1,1), \sigma_{1} \times \sigma_{2} \in \mathcal{P}_{s, t}\right\} \\
\geq\left\{\sup _{m \geq 1} \alpha_{s_{m}, t_{m}}\right\} \sup \left\{\frac{1}{\phi_{s_{m}, t_{m}}} \sum_{i \in \sigma_{1}} \sum_{j \in \sigma_{2}} \sum_{k, l=1}^{\infty} M_{k, l}^{\infty}\left(\left\|\frac{A_{i j}(x)}{\rho}, z_{1}, \ldots, z_{n-1}\right\|\right)^{p_{i j}}:\right. \\
\left.(s, t) \geq(1,1), \sigma_{1} \times \sigma_{2} \in \mathcal{P}_{s, t}\right\}=\infty .
\end{gathered}
$$


This is a contradiction as $x=\left\{x_{k, l}\right\} \notin m^{2}(\mathcal{M}, A, \phi, p,\|\cdot, \ldots, \cdot\|)$. This completes the proof of the theorem.

Corollary 2.4 Let $\phi$ and $\psi$ be two double sequences then $m^{2}(\mathcal{M}, A, \phi, p,\|\cdot, \ldots, \cdot\|)=$ $m^{2}(\mathcal{M}, A, \psi, p,\|\cdot, \ldots, \cdot\|)$ if and only if $\sup _{(s, t) \geq(1,1)} \alpha_{s, t}<\infty$ and $\sup _{(s, t) \geq(1,1)} \alpha_{s, t}^{-1}<\infty$.

Proof It is easy to prove so we omit the details.

Theorem 2.5 Let $\mathcal{M}=\left(M_{k, l}\right), \mathcal{M}^{\prime}=\left(M_{k, l}^{\prime}\right)$ and $\mathcal{M}^{\prime \prime}=\left(M_{k, l}^{\prime \prime}\right)$ be sequences of Orlicz functions satisfying $\Delta_{2}$-condition. Then

(i) $m^{2}(\mathcal{M}, \phi,\|\cdot, \ldots, \cdot\|) \subseteq m^{2}\left(\mathcal{M} \circ \mathcal{M}^{\prime}, \phi,\|\cdot, \ldots, \cdot\|\right)$,

(ii) $m^{2}\left(\mathcal{M}^{\prime}, A, \phi, p,\|\cdot, \ldots, \cdot\|\right) \cap m^{2}\left(\mathcal{M}^{\prime \prime}, A, \phi, p,\|\cdot, \ldots, \cdot\|\right) \subseteq$ $m^{2}\left(\mathcal{M}^{\prime}+\mathcal{M}^{\prime \prime}, A, \phi, p,\|\cdot, \ldots, \cdot\|\right)$.

Proof (i) Let $x=\left\{x_{k, l}\right\} \in m^{2}(\mathcal{M}, A, \phi, p,\|\cdot, \ldots, \cdot\|)$. Then there exists $\rho>0$ such that

$$
\sup \left\{\frac{1}{\phi_{s, t}} \sum_{i \in \sigma_{1}} \sum_{j \in \sigma_{2}} \sum_{k, l=1}^{\infty} M_{k, l}\left(\left\|\frac{A_{i j}(x)}{\rho}, z_{1}, \ldots, z_{n-1}\right\|\right)^{p_{i j}}:(s, t) \geq(1,1), \sigma_{1} \times \sigma_{2} \in \mathcal{P}_{s, t}\right\}<\infty .
$$

By the continuity of $\mathcal{M}$, we can take a number $\delta$ with $0<\delta<1$ such that $M_{k, l}(t)<\epsilon$, whenever $0 \leq t<\delta$, for arbitrary $0<\epsilon<1$. Now let

$$
y_{i, j}=\left(\left\|\frac{A_{i, j}(x)}{\rho}, z_{1}, \ldots, z_{n-1}\right\|\right) .
$$

Thus we have

$$
\sum_{i \in \sigma_{1}} \sum_{j \in \sigma_{2}} \sum_{k, l=1}^{\infty} M_{k, l}\left(y_{i, j}\right)^{p_{i, j}}=\sum_{y_{i, j} \leq \delta} \sum_{k, l=1}^{\infty} M_{k, l}\left(y_{i, j}\right)^{p_{i, j}}+\sum_{y_{i, j}>\delta} \sum_{k, l=1}^{\infty} M_{k, l}\left(y_{i, j}\right)^{p_{i, j}} .
$$

By the properties of the Orlicz function we have

$$
\sum_{y_{i, j} \leq \infty} \sum_{k, l=1}^{\infty} M_{k, l}\left(y_{i, j}\right)^{p_{i, j}} \leq \max \left\{1, M_{k, l}(1)^{H}\right\} \sum_{y_{i, j} \leq \infty}\left(y_{i, j}\right)^{p_{i, j}} .
$$

Again, we have

$$
M_{k, l}\left(y_{i, j}\right)<M_{k, l}\left(1+\frac{y_{i, j}}{\delta}\right)<\frac{1}{2} M_{k, l}(2)+\frac{1}{2} M_{k, l}\left(\frac{2 y_{i, j}}{\delta}\right)
$$

for $y_{i, j}>\delta$. If $\mathcal{M}$ satisfies the $\Delta_{2}$-condition, then we have

$$
M_{k, l}\left(y_{i, j}\right)<\frac{1}{2} T \frac{y_{i, j}}{\delta} M_{k, l}(2)+\frac{1}{2} T \frac{y_{i, j}}{\delta} M_{k, l}(2),
$$

and so

$$
\sum_{y_{i, j}>\delta} M_{k, l}\left(y_{i, j}\right)^{p_{i, j}} \leq \max \left(1,\left(\frac{T}{\delta} M_{k, l}(2)\right)^{H}\right) \sum_{y_{i, j}>\delta} y_{i, j} .
$$


Hence, we have

$$
\begin{aligned}
& \sup \left\{\frac{1}{\phi_{s, t}} \sum_{i \in \sigma_{1}} \sum_{j \in \sigma_{2}} \sum_{k, l=1}^{\infty} M_{k, l}\left(M_{k, l}^{\prime}\left(\left\|\frac{A_{i j}(x)}{\rho}, z_{1}, \ldots, z_{n-1}\right\|\right)\right)^{p_{i j}}:\right. \\
& \left.\quad(s, t) \geq(1,1), \sigma_{1} \times \sigma_{2} \in \mathcal{P}_{s, t}\right\} \\
& <\infty \\
& \leq \max \left\{1, M_{k, l}(1)^{H}\right\} \sup \left\{\frac{1}{\phi_{s, t}} \sum_{i \in \sigma_{1}} \sum_{j \in \sigma_{2}} \sum_{k, l=1}^{\infty} M_{k, l}\left(y_{i, j}\right)^{p_{i j}}:(s, t) \geq(1,1), \sigma_{1} \times \sigma_{2} \in \mathcal{P}_{s, t}\right\} \\
& <\infty+\max \left(1,\left(\frac{T}{\delta} M_{k, l}(2)\right)^{H}\right) \\
& \quad \times \sup \left\{\frac{1}{\phi_{s, t}} \sum_{i \in \sigma_{1}} \sum_{j \in \sigma_{2}} \sum_{k, l=1}^{\infty} M_{k, l}\left(y_{i, j}\right)^{p_{i j}}:(s, t) \geq(1,1), \sigma_{1} \times \sigma_{2} \in \mathcal{P}_{s, t}\right\}<\infty .
\end{aligned}
$$

Thus, we have $x=\left\{x_{k, l}\right\} \in m^{2}\left(\mathcal{M} \circ \mathcal{M}^{\prime}, \phi,\|\cdot, \ldots, \cdot\|\right)$ and hence $m^{2}(\mathcal{M}, \phi,\|\cdot, \ldots, \cdot\|) \subseteq$ $m^{2}\left(\mathcal{M} \circ \mathcal{M}^{\prime}, \phi,\|\cdot, \ldots, \cdot\|\right)$.

(ii) Let $x=\left\{x_{k, l}\right\} \in m^{2}\left(\mathcal{M}^{\prime}, A, \phi, p,\|\cdot, \ldots, \cdot\|\right) \cap m^{2}\left(\mathcal{M}^{\prime \prime}, A, \phi, p,\|\cdot, \ldots, \cdot\|\right)$. Then there exists a $\rho>0$ such that

$$
\sup \left\{\frac{1}{\phi_{s, t}} \sum_{i \in \sigma_{1}} \sum_{j \in \sigma_{2}} \sum_{k, l=1}^{\infty} M_{k, l}^{\prime}\left(\left\|\frac{A_{i, j}(x)}{\rho}, z_{1}, \ldots, z_{n-1}\right\|\right)^{p_{i j}}:(s, t) \geq(1,1), \sigma_{1} \times \sigma_{2} \in \mathcal{P}_{s, t}\right\}<\infty
$$

and

$$
\sup \left\{\frac{1}{\phi_{s, t}} \sum_{i \in \sigma_{1}} \sum_{j \in \sigma_{2}} \sum_{k, l=1}^{\infty} M_{k, l}^{\prime \prime}\left(\left\|\frac{A_{i, j}(x)}{\rho}, z_{1}, \ldots, z_{n-1}\right\|\right)^{p_{i j}}:(s, t) \geq(1,1), \sigma_{1} \times \sigma_{2} \in \mathcal{P}_{s, t}\right\}<\infty
$$

By the inequality, we have

$$
\begin{aligned}
& \sum_{i \in \sigma_{1}} \sum_{j \in \sigma_{2}} \sum_{k, l=1}^{\infty}\left(M_{k, l}^{\prime}+M_{k, l}^{\prime \prime}\right)\left(\left\|\frac{A_{i, j}(x)}{\rho}, z_{1}, \ldots, z_{n-1}\right\|\right)^{p_{i j}} \\
& \leq \max \left(1,2^{H-1}\right) \sum_{i \in \sigma_{1}} \sum_{j \in \sigma_{2}} \sum_{k, l=1}^{\infty} M_{k, l}^{\prime}\left(\left\|\frac{A_{i, j}(x)}{\rho}, z_{1}, \ldots, z_{n-1}\right\|\right)^{p_{i j}} \\
& \quad+\max \left(1,2^{H-1}\right) \sum_{i \in \sigma_{1}} \sum_{j \in \sigma_{2}} \sum_{k, l=1}^{\infty} M_{k, l}^{\prime \prime}\left(\left\|\frac{A_{i, j}(x)}{\rho}, z_{1}, \ldots, z_{n-1}\right\|\right)^{p_{i j}} .
\end{aligned}
$$

Hence

$$
\begin{aligned}
& m^{2}\left(\mathcal{M}^{\prime}, A, \phi, p,\|\cdot, \ldots, \cdot\|\right) \cap m^{2}\left(\mathcal{M}^{\prime \prime}, A, \phi, p,\|\cdot, \ldots, \cdot\|\right) \\
& \quad \subseteq m^{2}\left(\mathcal{M}^{\prime}+\mathcal{M}^{\prime \prime}, A, \phi, p,\|\cdot, \ldots, \cdot\|\right) .
\end{aligned}
$$

This completes the proof of the theorem. 
Theorem 2.6 The sequence space $m^{2}(\mathcal{M}, \phi, p,\|\cdot, \ldots, \cdot\|)$ is solid.

Proof Let $\alpha=\left\{\alpha_{k, l}\right\}$ be a double sequence of scalars such that $\left|\alpha_{k, l}\right| \leq 1$ and $y=\left\{y_{k, l}\right\} \in$ $m^{2}(\mathcal{M}, \phi, p,\|\cdot, \ldots, \cdot\|)$. Then we have

$$
\begin{aligned}
& \sup \left\{\frac{1}{\phi_{s, t}} \sum_{k \in \sigma_{1}} \sum_{l \in \sigma_{2}} M_{k, l}\left(\left\|\frac{\alpha_{k, l} x_{k, l}}{\rho}, z_{1}, \ldots, z_{n-1}\right\|\right)^{p_{i j}}:(s, t) \geq(1,1), \sigma_{1} \times \sigma_{2} \in \mathcal{P}_{s, t}\right\} \\
& \quad \leq \sup \left\{\frac{1}{\phi_{s, t}} \sum_{k \in \sigma_{1}} \sum_{l \in \sigma_{2}} M_{k, l}\left(\left\|\frac{\alpha_{k, l} y_{k, l}}{\rho}, z_{1}, \ldots, z_{n-1}\right\|\right)^{p_{i j}}:(s, t) \geq(1,1), \sigma_{1} \times \sigma_{2} \in \mathcal{P}_{s, t}\right\} \\
& \quad \leq \sup \left\{\frac{1}{\phi_{s, t}} \sum_{k \in \sigma_{1}} \sum_{l \in \sigma_{2}} M_{k, l}\left(\left\|\frac{y_{k, l}}{\rho}, z_{1}, \ldots, z_{n-1}\right\|\right)^{p_{i j}}:(s, t) \geq(1,1), \sigma_{1} \times \sigma_{2} \in \mathcal{P}_{s, t}\right\} .
\end{aligned}
$$

This implies that $\left\{\alpha_{k, l} y_{k, l}\right\} \in m^{2}(\mathcal{M}, \phi, p,\|\cdot, \ldots, \cdot\|)$. This proves that the space $m^{2}(\mathcal{M}, \phi, p$, $\|\cdot, \ldots, \cdot\|)$ is a solid.

Corollary 2.7 The sequence space $m^{2}(\mathcal{M}, \phi, p,\|\cdot, \ldots, \cdot\|)$ is monotone.

Proof It is trivial so we omit the details.

\section{Competing interests}

The authors declare that they have no competing interests.

Authors' contributions

All authors contributed equally and significantly in writing this paper. All authors read and approved the final manuscript.

\section{Author details}

'Department of Mathematics, Faculty of Science, King Abdulaziz University, P.O. Box 80203, Jeddah, 21589, Saudi Arabia. ${ }^{2}$ Department of Mathematics, Aligarh Muslim University, Aligarh, 202002, India. ${ }^{3}$ Department of Mathematics, Model

Institute of Engineering and Technology, Kot Bhalwal, JK 181122, India.

\section{Received: 21 February 2014 Accepted: 9 May 2014 Published: 29 May 2014}

\section{References}

1. Bromwich, TJ: An Introduction to the Theory of Infinite Series. Macmillan Co., New York (1965)

2. Hardy, GH: On the convergence of certain multiple series. Proc. Camb. Philos. Soc. 19, 86-95 (1917)

3. Moricz, F: Extension of the spaces $c$ and $c_{0}$ from single to double sequences. Acta Math. Hung. 57, 129-136 (1991)

4. Moricz, F, Rhoades, BE: Almost convergence of double sequences and strong regularity of summability matrices. Math. Proc. Camb. Philos. Soc. 104, 283-294 (1988)

5. Başarır, M, Sonalcan, O: On some double sequence spaces. J. Indian Acad. Math. 21, 193-200 (1999)

6. Zeltser, M: Investigation of Double Sequence Spaces by Soft and Hard Analytical Methods. Diss. Math. Univ. Tartu, vol. 25. Tartu University Press, Tartu (2001)

7. Mursaleen, M, Edely, OHH: Statistical convergence of double sequences. J. Math. Anal. Appl. 288(1), 223-231 (2003)

8. Mohiuddine, SA, Alotaibi, A, Mursaleen, M: Statistical convergence of double sequences in locally solid Riesz spaces. Abstr. Appl. Anal. 2012, Article ID 719729 (2012). doi:10.1155/2012/719729

9. Mursaleen, M: Almost strongly regular matrices and a core theorem for double sequences. J. Math. Anal. Appl. 293(2), 523-531 (2004)

10. Mursaleen, M, Savas, E: Almost regular matrices for double sequences. Studia Sci. Math. Hung. 40, 205-212 (2003)

11. Altay, B, Başar, F: Some new spaces of double sequences. J. Math. Anal. Appl. 309, 70-90 (2005)

12. Başar, F, Sever, Y: The space $\mathcal{L}_{a}$ of double sequences. Math. J. Okayama Univ. 51, 149-157 (2009)

13. Raj, K, Sharma, SK: Some multiplier double sequence spaces. Acta Math. Vietnam. 37, 391-406 (2012)

14. Pringsheim, A: Zur Theorie der zweifach unendlichen Zahlenfolgen. Math. Ann. 53, 289-321 (1900)

15. Limaye, BV, Zeltser, M: On the Pringsheim convergence of double series. Proc. Est. Acad. Sci. 58, 108-121 (2009)

16. Cakan, C, Altay, B, Mursaleen, M: The $\sigma$-convergence and $\sigma$-core of double sequences. Appl. Math. Lett. 19 $1122-1128(2006)$

17. Mursaleen, M, Mohiuddine, SA: Double $\sigma$-multiplicative matrices. J. Math. Anal. Appl. 327, $991-996$ (2007)

18. Mursaleen, M, Mohiuddine, SA: Regularly $\sigma$-conservative and $\sigma$-coercive four dimensional matrices. Comput. Math. Appl. 56, 1580-1586 (2008)

19. Mursaleen, M, Mohiuddine, SA: On $\sigma$-conservative and boundedly $\sigma$-conservative four dimensional matrices. Comput. Math. Appl. 59, 880-885 (2010) 
20. Mursaleen, M, Mohiuddine, SA: Convergence Methods for Double Sequences and Applications. Springer, Berlin (2014)

21. Mohiuddine, SA, Alotaibi, A: Some spaces of double sequences obtained through invariant mean and related concepts. Abstr. Appl. Anal. 2013, Article ID 507950 (2013)

22. Mohiuddine, SA, Raj, K, Alotaibi, A: Some paranormed double difference sequence spaces for Orlicz functions and bounded-regular matrices. Abstr. Appl. Anal. 2014, Article ID 419064 (2014)

23. Sharma, SK, Raj, K, Sharma, AK: Some new double sequence spaces over n-normed space. Int. J. Appl. Math. 25, 255-269 (2012)

24. Lindenstrauss, J, Tzafriri, L: On Orlicz sequence spaces. Isr. J. Math. 10, 345-355 (1971)

25. Musielak, J: Orlicz Spaces and Modular Spaces. Lecture Notes in Mathematics, vol. 1034 (1983)

26. Maligranda, L: Orlicz Spaces and Interpolation. Seminars in Mathematics, vol. 5. Polish Academy of Science, Warsaw (1989)

27. Raj, K, Sharma, SK: Some multiplier sequence spaces defined by a Musielak-Orlicz function in $n$-normed spaces. N.Z. J. Math. 42, 45-56 (2012)

28. Raj, K, Sharma, SK: Some double sequence spaces defined by a sequence of Orlicz function. J. Math. Anal. 3, 12-20 (2012)

29. Raj, K, Sharma, SK: Some generalized difference double sequence spaces defined by a sequence of Orlicz-function. CUBO 14, 167-189 (2012)

30. Wilansky, A: Summability Through Functional Analysis. North-Holland Math. Stud., vol. 85 (1984)

31. Gähler, S: Lineare 2-normierte Räume. Math. Nachr. 28, 1-43 (1965)

32. Misiak, A: $n$-Inner product spaces. Math. Nachr. 140, 299-319 (1989)

33. Gunawan, H: On n-inner product, n-norms, and the Cauchy-Schwartz inequality. Sci. Math. Jpn. 5, 47-54 (2001)

34. Gunawan, H: The space of $p$-summable sequence and its natural n-norm. Bull. Aust. Math. Soc. 64, 137-147 (2001)

35. Gunawan, H, Mashadi, M: On n-normed spaces. Int. J. Math. Math. Sci. 27, 631-639 (2001)

36. Sargent, WLC: Some sequence spaces related to the $I_{p}$ spaces. J. Lond. Math. Soc. 35, 161-171 (1960)

37. Malkowsky, E, Mursaleen, M: Matrix transformations between FK-spaces and the sequence spaces $m(\phi)$ and $n(\phi)$. J. Math. Anal. Appl. 196, 659-665 (1995)

38. Tripathy, BC, Sen, M: On a new class of sequences related to the space $I_{p}$. Tamkang J. Math. 33, 167-171 (2002)

39. Mursaleen, M: On some geometric properties of a sequence space related to $\ell_{p}$. Bull. Aust. Math. Soc. 67, 343-347 (2003)

40. Duyar, $C$, Oǧur, O: On a new space $m^{2}(M, A, \phi, p)$ of double sequences. J. Funct. Spaces Appl. 2013, Article ID 509613 (2013)

10.1186/1029-242X-2014-216

Cite this article as: Alotaibi et al.: Double sequence spaces over $n$-normed spaces defined by a sequence of Orlicz functions. Journal of Inequalities and Applications 2014, 2014:216

\section{Submit your manuscript to a SpringerOpen ${ }^{\circ}$ journal and benefit from:}

- Convenient online submission

Rigorous peer review

- Immediate publication on acceptance

- Open access: articles freely available online

- High visibility within the field

- Retaining the copyright to your article 